\title{
Emprego da Ventilação Mecânica com Pressão Controlada em Circuito Circular de Anestesia para Pacientes de Baixo Peso: Estudo Experimental *
}

\section{Controlled Pressure Mechanical Ventilation with Anesthesia Closed System for Low Weight Patients: Experimental Study}

Denise Tabacchi Fantoni ${ }^{1}$, Sandra Mastrocinque ${ }^{2}$, Silvia Renata Gaido Cortopassi ${ }^{3}$, Elton R. Migliatti ${ }^{2}$, José Otávio Costa Auler Junior, TSA ${ }^{4}$

\section{RESUMO}

Fantoni DT, Mastrocinque S, Cortopassi SRG, Migliatti ER, Auler Jr JOC - Emprego da Ventilação Mecânica com Pressão Controlada em Circuito Circular de Anestesia para Pacientes de Baixo Peso: Estudo Experimental

Justificativa e Objetivos - $A$ anestesia com baixo fluxo, em pacientes pediátricos, requer equipamentos adequados, no entanto, os disponíveis no mercado são de alto custo, o que limita o seu uso. Este estudo avaliou a anestesia com baixo fluxo em coelhos, empregando circuito fechado, modo de pressão controlada em novo ventilador pediátrico para anestesia (VPL-5000A-Vent-Logos).

Método - Dez coelhos foram distribuídos aleatoriamente, sendo que o grupo I foi submetido à pressão de $15 \mathrm{cmH}_{2} \mathrm{O}$ e o grupo II à de $20 \mathrm{cmH}_{2} \mathrm{O}$. A anestesia foi realizada com xilazina (10 mg. $\left.\mathrm{kg}^{-1}\right)$ e cetamina (25 mg. $\mathrm{kg}^{-1}$ ) associados, por via muscular, seguida de manutenção com isoflurano, após intubação orotraqueal. Após 20 minutos, administrou-se pancurônio $(0,1$ $\mathrm{mg} \cdot \mathrm{kg}^{-1}$ ) por via venosa e a ventilação controlada foi iniciada. Os parâmetros ajustados no ventilador foram: FR $30 \mathrm{mpm}$, freqüência I:E 1:2, 5 e tempo de inspiração 0,6 segundo, além das pressões de plateau. O fluxo de gases frescos empregado foi $300 \mathrm{ml}$ (total). Os parâmetros foram coletados a cada 20 minutos durante uma hora. Os dados obtidos foram submetidos à análise estatística de variância para medidas repetidas ( $p<$ $0,05)$.

Resultados - $\mathrm{O} \mathrm{CO}_{2}$ reinalado diminuiu significativamente no grupo II, de $15 \mathrm{mmHg}$, durante a ventilação espontânea, para um valor médio de $2,4 \mathrm{mmHg}$, durante a ventilação controlada. No grupo I, diminuiu de 19,2 $\mathrm{mmHg}$ (inicial) para 3,6 $\mathrm{mmHg}$. Comparando-se os dois grupos, diferenças significativas foram encontradas em relação ao $\mathrm{pH}$ venoso, $\mathrm{PaCO}_{2}, \mathrm{PvO}_{2}$ e discreta diferença entre a PAM e PAD. O grupo de $15 \mathrm{cmH}_{2} \mathrm{O}$ apresentou importante acidose respiratória, enquanto o de 20 $\mathrm{CmH}_{2} \mathrm{O}$ obteve valores normais de $\mathrm{pH}$ e $\mathrm{PaCO}_{2}$. Uma vez que os valores de volume expirado entre os grupos foram semelhantes, tais diferenças entre $\mathrm{pH}$ e gases sangüíneos

\footnotetext{
* Recebido do (Received from) Laboratório de Anestesiologia da Faculdade de Medicina Veterinária e Zootecnia da Universidade de São Paulo

1. Médica Veterinária, Professora Livre Docente, FMVZ-USP

2. Médico Veterinário, Aluno de Mestrado em Cirurgia, FMVZ-USP

3. Médica Veterinária, Professora Doutora, FMVZ-USP

4. Professor Titular da Disciplina de Anestesiologia, FM-USP

Apresentado (Submitted) em 29 de setembro de 2000

Aceito (Accepted) para publicação em 02 de março de 2001

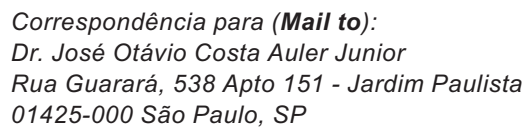

(C) Sociedade Brasileira de Anestesiologia, 2001
}

apresentados pelos grupos podem estar relacionadas aos baixos níveis de $\mathrm{pH}$ observados no grupo I. Verificou-se consumo médio de $2 \mathrm{ml}$ de isoflurano por animal durante os 120 minutos de estudo.

Conclusões - Com equipamento adequado é possível empregar anestesia de baixo fluxo, ventilação com pressão controlada e circuito fechado em pacientes com peso muito baixo.

UNITERMOS - ANIMAL: coelho; EQUIPAMENTOS: sistema respiratório, circuito fechado; VENTILAÇÃO: mecânica

\section{SUMMARY}

Fantoni DT, Mastrocinque S, Cortopassi SRG, Migliatti ER, Auler Jr JOC - Controlled Pressure Mechanical Ventilation with Anesthesia Closed System for Low Weight Patients: Experimental Study

Background and Objectives - Pediatric low flow anesthesia requires adequate equipment which, when available, is extremely expensive, thus seldom used. This study aimed at evaluating low flow anesthesia in rabbits using a closed rebreathing circuit in a new pediatric pressure controlled ventilator for anesthesia.

Methods - Ten rabbits were randomly assigned to two groups. Group I individuals were ventilated with the airway pressure limit set to $15 \mathrm{cmH}_{2} \mathrm{O}$, while in group II the setting was $20 \mathrm{~cm}$ $\mathrm{H}_{2} \mathrm{O}$. Anesthesia was induced with muscular xylazine $(10$ $\mathrm{mg} \cdot \mathrm{kg}^{-1}$ ) and ketamine (25 mg. $\left.\mathrm{kg}^{-1}\right)$, followed by maintenance with isoflurane after tracheal intubation. After 20 minutes, 0.1 $\mathrm{mg} . \mathrm{kg}^{-1}$ intravenous pancuronium was administered and controlled ventilation was established. Ventilator parameters were: $R R$ - $30 \mathrm{mpm}$, I:E ratio 1:2.5 and inspiratory time $0.6 \mathrm{sec}$, in addition to plateau pressures. Fresh gas flow was $300 \mathrm{ml}^{.} \mathrm{min}^{-1}$ (total). Parameters were collected every 20 minutes for one hour and data were submitted to analysis of variance for repeated measures $(p<0.05)$.

Results - Re-inhaled $\mathrm{CO}_{2}$ decreased significantly in group II from an initial value of $15 \mathrm{mmHg}$ during spontaneous ventilation to a mean value of $2.4 \mathrm{mmHg}$ during controlled ventilation. In group I, the drop was from $19.2 \mathrm{mmHg}$ (initial) to $3.6 \mathrm{mmHg}$. Comparing both groups, significant differences were observed in venous $\mathrm{pH}, \mathrm{PaCO}_{2}, \mathrm{PvO}_{2}$ and a slight difference between $\mathrm{MBP}$ and $D B P$. The $15 \mathrm{cmH}_{2} \mathrm{O}$ group showed important respiratory acidosis, while the $20 \mathrm{cmH}_{2} \mathrm{O}$ had normal $\mathrm{pH}$ and $\mathrm{PaCO}_{2}$ values. Since expired volume values were similar in both groups, such differences in $\mathrm{pH}$ and blood gases observed could be related to low $\mathrm{pH}$ levels seen in group I. Each animal consumed a mean value of $2 \mathrm{ml}$ isoflurane during the 120 minutes of the study.

Conclusions - With proper equipment, it is possible to use low flow anesthesia with pressure controlled ventilation and closed system in very low weight patients.

KEY WORDS - ANIMAL: rabbits; EQUIPMENTS: respiratory system, closed circuit; VENTILATION: mechanical 


\section{INTRODUÇÃO}

Nos os últimos anos, tem surgido interesse crescente no emprego de circuitos circulares em crianças. O uso destes circuitos, associados a ventiladores de alta precisão, permite a utilização de fluxos menores de gases frescos, melhor conservação de umidade e calor e, sem dúvida alguma, menor consumo de anestésico e poluição do centro cirúrgico ${ }^{1}$. Outro grande benefício é a possibilidade de utilização de diferentes modalidades ventilatórias e recursos, como pressão expiratória final positiva (PEEP), presentes nestes equipamentos que, certamente, são necessários nos pacientes de alto risco. No entanto, em nosso meio, a anestesia do paciente pediátrico tem sido um desafio para o anestesiologista devido à falta de equipamento adequado. Em muitos centros, os circuitos sem reinalação, como os circuitos de Bain ou Mapleson D, são ainda amplamente usados em pediatria, sendo a ventilação controlada realizada manualmente. Nestes circuitos, fluxos altos de gases frescos são empregados, o que acarreta grande consumo de anestésico e poluição do ambiente. Outra grande desvantagem desta prática é a falta de monitorização precisa de parâmetros, como volume expirado, pressão de vias aéreas, entre outros.

Um dos grandes empecilhos para o emprego dos ventiladores e circuitos circulares de anestesia, disponíveis para adultos e que podem ser empregados nos pacientes de baixo peso, é o custo elevado dos mesmos. Também a possibilidade de realização da anestesia com baixo fluxo em crianças, seria outra grande vantagem dos novos equipamentos disponíveis no mercado.

O objetivo do presente estudo foi avaliar um equipamento de anestesia com circuito circular fechado, utilizando modo ventilatório ciclado a tempo com pressão-controlada e emprego de baixo fluxo de gases frescos. Para isto, utilizou-se animais de pequeno porte (coelhos), no intuito de se verificar a eficácia deste equipamento na ventilação durante a anestesia.

\section{MÉTODO}

Foram utilizados 10 coelhos, machos e fêmeas, pesando entre 3,5 e $5 \mathrm{~kg}$. A anestesia foi realizada com o emprego de xilazina $\left(10 \mathrm{mg} \cdot \mathrm{kg}^{-1}\right)$ e cetamina $\left(25 \mathrm{mg} \cdot \mathrm{kg}^{-1}\right)$ administrados por via muscular e para indução anestésica empregou-se o isoflurano via máscara. Procedeu-se à intubação orotraqueal com sonda de Magill com balonete (2,5 a 3,0 mm de diâmetro interno). A anestesia foi mantida com isoflurano em $100 \%$ de oxigênio. Durante 20 minutos os animais permaneceram em ventilação espontânea até a estabilização no equipamento de anestesia. Aartéria marginal e veia da orelha foram cateterizadas para monitorização da pressão arterial e administração de fluidoterapia (Ringer com lactato, $2 \mathrm{ml} . \mathrm{kg} \cdot \mathrm{h}^{-1}$ ) e fármacos, respectivamente. Em seguida, administrou-se pancurônio $\left(0,1 \mathrm{mg} \cdot \mathrm{kg}^{-1}\right)$ e iniciou-se a ventilação controlada por período de 60 minutos, realizada no modo ciclado a tem- po na modalidade pressão controlada. Os parâmetros ajustados no ventilador foram: freqüência respiratória de 30 movimentos por minuto, relação I:E de 1:2,5 e tempo inspiratório de 0,6 segundos. O fluxo de gases frescos empregado foi de $300 \mathrm{ml}$. $\mathrm{min}^{-1}$ em fluxômetro não calibrado por pressão. Os animais foram então distribuídos aleatoriamente em dois grupos de cinco animais cada. No grupo I (G15), a pressão de pico empregada foi de $15 \mathrm{cmH}_{2} \mathrm{O}$, enquanto que no grupo II (G20) empregou-se $20 \mathrm{cmH}_{2} \mathrm{O}$. O fluxo de gás ajustado no ventilador foi aquele suficiente para gerar curva de pressão quadrada condizente com a modalidade desejada.

Para avaliação da adequação da ventilação mensurou-se de forma contínua a concentração de dióxido de carbono no ar expirado, $a \mathrm{FiO}_{2}$, a saturação periférica de oxigênio da hemoglobina, realizando-se ainda exames seriados do $\mathrm{pH}$, gases sangüíneos e bicarbonato plasmático.

Através de monitor de ventilação, mensurou-se também o volume inspirado e expirado, o volume minuto, pressão de pico, platô e média, bem como a complacência pulmonar. Este monitor assegurou a modalidade respiratória empregada através da visualização das curvas de pressão. Para monitorização da anestesia avaliou-se, continuamente, a concentração inspirada e expirada de isoflurano, o eletrocardiograma e a pressão arterial.

Para este estudo, utilizou-se um sistema pneumático de controle de fases inspiratória e expiratória, ciclado a tempo, que apresenta como controles: fluxo, tempo inspiratório, tempo expiratório, pressão máxima e PEEP, além de um alarme que detecta desconexão ou ausência de ciclo.

O sistema está formado por quatro subconjuntos (Figura 1):

1) Um automático de ciclagem a tempo com limite de pressão;

2) Um sistema tipo "Bag in a Bottle";

3) Um conjunto formado por uma combinação de válvulas que permite ao aparelho funcionar com respiração controlada (mecânica ou manual) ou espontânea, e;

4) Um reservatório de absorvedor de gás carbônico.

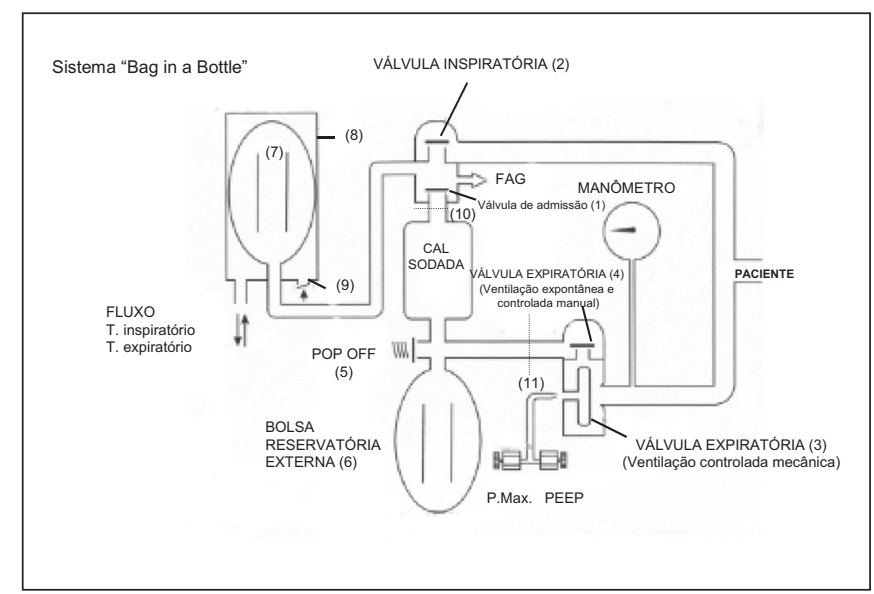

Figura 1 - Sistema Utilizado no Experimento

Revista Brasileira de Anestesiologia Vol. 51, № 4, Julho - Agosto, 2001 
Durante a fase expiratória uma pressão subatmosférica age entre a bolsa reservatório interna (7) e a campânula (8), permitindo que ela se encha da mistura gasosa, após passar pela válvula unidirecional (1). Uma outra válvula unidirecional (2) impede que o volume expirado pelo paciente retorne à bolsa (7).

Durante a fase inspiratória, um volume de gás (gerado pelo fluxo no tempo inspiratório) comprime a bolsa reservatório interna (7). A velocidade desta compressão é proporcional ao ajuste do fluxo, que deve mimetizar uma compressão manual. A válvula unidirecional (1) fecha e a válvula (2) se abre, permitindo que o conteúdo da bolsa (7) se dirija ao paciente. Uma válvula expiratória (3) sustenta o volume corrente até que a pressão da via aérea, previamente ajustada (pressão inspiratória máxima), seja atingida.

A partir deste ponto, o volume excedido escapa, abrindo e ultrapassando a válvula unidirecional (4), e pode se dirigir:

a) para a bolsa reservatório externa (6);

b) para a atmosfera ou sistema anti-poluição, se a bolsa reservatório externa (6) estiver cheia, através da válvula pop-off (5);

c) diretamente para a atmosfera se o sistema estiver aberto.

A colocação de um fluxo adicional de gases (FAG) entre as válvulas (1)e (2) permite que a segunda se mantenha aberta, qualquer que seja o FAG e desde que a bolsa reservatório interna (7) se encontre cheia. Essa particularidade permite ao pequeno paciente ventilar sem resistência.

Durante a fase expiratória, a bolsa reservatório interna (7) se enche, captando a mistura gasosa proveniente da bolsa reservatório externa (6), bem como do volume expirado pelo paciente (após passar pelo absorvedor de gás carbônico). Se o sistema for sem absorção de gás carbônico, ocorre a aspiração de ar da atmosfera.

A válvula (9) tem como finalidade conectar a parede externa da bolsa reservatório interna (7) com a atmosfera e isso acontece com o aparelho desligado (respiração espontânea e controlada manual). Esta, também serve como válvula de segurança do sistema "Bag in a Bottle", não permitindo que a pressão neste ponto ultrapasse a $80 \mathrm{cmH}_{2} \mathrm{O}$.

Para se medir, corretamente, a pressão de boca aproveitou-se o ramo expiratório, sendo a pressão medida antes da válvula (3). Esta medida não aufere a pressão resistiva da passagem do fluxo pela via inspiratória.

O sistema transforma-se em "sem absorção" de gás carbônico no momento em que um ou os dois tubos corrugados (10 e 11), que conectam o ventilador ao sistema absorvedor, forem desconectados. Este procedimento poderá ser realizado quando se desejar um despertar mais rápido.

O aparelho permite a ventilação mandatória intermitente, para tanto basta aumentar o tempo expiratório.

Os resultados obtidos foram analisados através de programa computacional (INSTAT), sendo os dados submetidos à análise de variância ANOVA e empregado-se o tese $t$ de Student para comparação das médias dos diferentes grupos avaliados. O nível de significância estabelecido para os testes realizados foi de $5 \%$.

\section{RESULTADOS}

Os resultados obtidos nos dois grupos experimentais estão apresentados nas tabelas I, II e III. Não foi verificada diferença significativa entre os grupos experimentais no que alude aos valores obtidos no momento controle e os da ventilação espontânea.

Durante a ventilação espontânea, em ambos os grupos, verificou-se altos valores de dióxido de carbono inspirado e expirado. Com a instituição da ventilação controlada, verificou-se incremento da ventilação, fato constatado através da normalização do $\mathrm{P}_{\mathrm{ET}} \mathrm{CO}_{2}$. $\mathrm{O} \mathrm{CO}_{2}$ inspirado diminuiu, mantendo-se em valores compatíveis com a adequação da ventilação. As pressões das vias aéreas e os volumes inspirado e expirado sofreram aumentos significativos durante a ventilação controlada em relação à ventilação espontânea.

$\mathrm{Na}$ análise comparativa realizada entre os dois grupos experimentais, os únicos valores que diferiram de forma significativa foram aqueles referentes à $\mathrm{PaCO}_{2}, \mathrm{PvO}_{2}$ e $\mathrm{PvCO}_{2}$, bem como o $\mathrm{pH}$ venoso. O grupo ventilado com $20 \mathrm{cmH}_{2} \mathrm{O}$ apresentou os menores valores da $\mathrm{PaCO}_{2}$ (Figura 2) e $\mathrm{PvCO}_{2}$ eos maiores valores da $\mathrm{PvO}_{2}$ e $\mathrm{pH}$. No que concerne aos valores da freqüência cardíaca e pressão arterial, não foram verificadas diferenças significativas entre os dois grupos ou entre as avaliações realizadas previamente à instituição de ventilação controlada com aquelas obtidas após seu início.

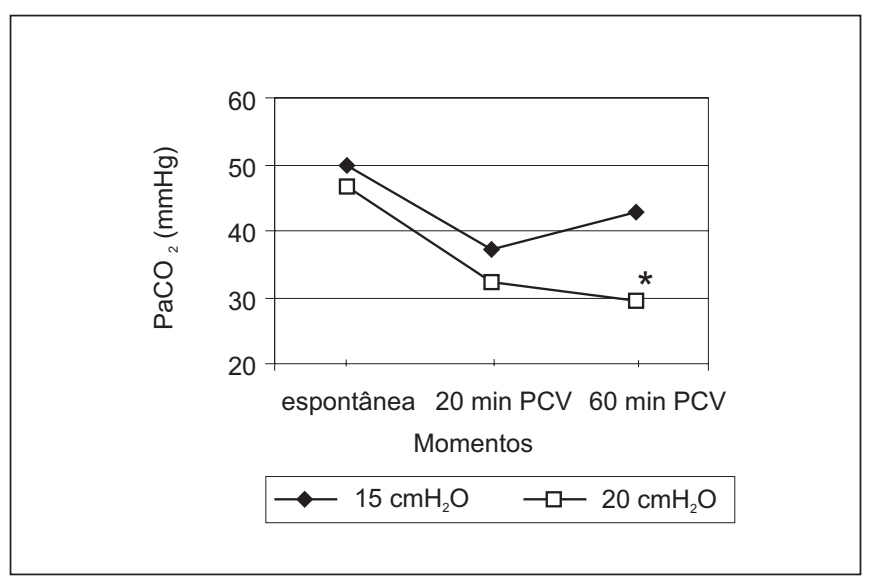

Figura 2 - Valores de Pressão Parcial de Dióxido de Carbono Arterial $(\mathrm{mmHg})$ nos Momentos de Ventilação Espontânea, aos 20 e aos 60 Minutos de Ventilação Controlada nos Grupos Ventilados com15 $\mathrm{cmH}_{2} \mathrm{O}$ ou $20 \mathrm{cmH}_{2} \mathrm{O}$

* difere de forma significativa do grupo ventilado com $15 \mathrm{cmH}_{2} \mathrm{O}$

Durante o tempo de avaliação da ventilação controlada, o consumo de isoflurano foi de $2 \mathrm{ml}$ por animal.

Após 90 minutos de anestesia, o isoflurano foi descontinuado, os animais extubados, não sendo observadas intercorrências. 
Tabela I - Parâmetros da Ventilação, Oxigenação e pH dos Animais Submetidos à Pressão Inspiratória de 15 (GI) ou 20 $\mathrm{cmH}_{2} \mathrm{O}$ (GII) em Circuito com Reinalação de Anestesia em Diferentes Tempos de Avaliação

\begin{tabular}{|c|c|c|c|c|c|c|}
\hline Parâmetro & Grupo & Controle & Espontânea & $20 \min P C V$ & $40 \mathrm{~min}$ PCV & $60 \mathrm{~min} P C V$ \\
\hline \multirow[t]{2}{*}{$\mathrm{CO}_{2}$ inspirado $(\mathrm{mmHg})$} & GI & $19,2 \pm 6,38$ & $24,0 \pm 6,42$ & $3,6 \pm 1,34^{*} \#$ & $3,6 \pm 0,89^{*}$ & $4,4 \pm 1,6^{*}$ \\
\hline & GII & $15,0 \pm 4,41$ & $16,2 \pm 3,83$ & $2,6 \pm 2,07^{*}$ & $2,4 \pm 1,67^{*}$ & $2,4 \pm 1,67^{\star}$ \\
\hline \multirow{2}{*}{$\mathrm{P}_{\mathrm{ET}} \mathrm{CO}_{2}(\mathrm{mmHg})$} & GI & $48,0 \pm 8,42$ & $52,2 \pm 8,34$ & $40,6 \pm 6,38$ & $37,0 \pm 5,24$ & $44,0 \pm 5,98$ \\
\hline & GII & $49,4 \pm 6,50$ & $48,4 \pm 7,95$ & $33,6 \pm 9,37$ & $32,0 \pm 8,63^{*}$ & $33,0 \pm 9,24$ \\
\hline \multirow[t]{2}{*}{$\mathrm{PH}$} & $\mathrm{Gl}$ & & $7,30 \pm 0,12$ & $7,34 \pm 0,07$ & & $7,33 \pm 0,12$ \\
\hline & GII & & $7,38 \pm 0,09$ & $7,45 \pm 0,06$ & & $7,43 \pm 0,06$ \\
\hline \multirow[t]{2}{*}{$\mathrm{PaCO}_{2}(\mathrm{mmHg})$} & GI & & $50,0 \pm 9,92$ & $37,2 \pm 8,5$ & & $42,8 \pm 18,58$ \\
\hline & GII & & $46,6 \pm 9,6$ & $32,2 \pm 5,8^{*}$ & & $29,4 \pm 4,72^{*}$ \\
\hline \multirow[t]{2}{*}{$\mathrm{PaO}_{2}(\mathrm{mmHg})$} & $\mathrm{GI}$ & & $295,4 \pm 49,8$ & $267,8 \pm 103,8$ & & $256,4 \pm 64,86$ \\
\hline & GII & & $306,8 \pm 81,51$ & $216,6 \pm 91,1$ & & $286,0 \pm 112,8$ \\
\hline \multirow[t]{2}{*}{$\mathrm{HCO}_{3}$ (mEq.L) } & GI & $24,0 \pm 7,17$ & & $20,0 \pm 7,41$ & & $21,2 \pm 7,79$ \\
\hline & GII & $25,8 \pm 4,08$ & & $22,0 \pm 4,84$ & & $21,4 \pm 5,59$ \\
\hline \multirow[t]{2}{*}{$\mathrm{PH}$ venoso } & GI & $7,30 \pm 0,07$ & & $7,31 \pm 0,06$ & & $7,29 \pm 0,13$ \\
\hline & GII & $7,34 \pm 0,06$ & & $7,34 \pm 0,12$ & & $7,39 \pm 0,03$ \\
\hline \multirow[t]{2}{*}{$\mathrm{PvO}_{2}(\mathrm{mmHg})$} & GI & $74,0 \pm 44,4$ & & $64,2 \pm 16,7$ & & $53,6 \pm 5,68$ \\
\hline & GII & $61,0 \pm 18,66$ & & $72,0 \pm 42,98$ & & $63,6 \pm 37,46$ \\
\hline \multirow[t]{2}{*}{$\mathrm{PvCO}_{2}(\mathrm{mmHg})$} & $\mathrm{Gl}$ & $44,4 \pm 17,37$ & & $34,2 \pm 10,2$ & & $44,4 \pm 21,19$ \\
\hline & GII & $36,0 \pm 14,0$ & & $29,4 \pm 5,45$ & & $31,0 \pm 2,34$ \\
\hline \multirow[t]{2}{*}{$\mathrm{SvO}_{2}(\%)$} & GI & $82,0 \pm 6,55$ & & $90,2 \pm 9,14$ & & $76,0 \pm 5,33$ \\
\hline & GII & $84,8 \pm 7,82$ & & $83,0 \pm 13,05$ & & $79,6 \pm 11,30$ \\
\hline
\end{tabular}

* difere significativamente do valor da ventilação espontânea sendo $p<0,05$; \# difere significativamente do G20 sendo $p<0,05$; Valores expressos em Média \pm DP

Tabela II - Parâmetros Cardiovasculares e da Anestesia dos Animais Submetidos à Pressão Inspiratória de 15 (GI) ou 20 $\mathrm{cmH}_{2} \mathrm{O}$ (GII) em Circuito com Reinalação de Anestesia em Diferentes Tempos de Avaliação

\begin{tabular}{lcccccc}
\hline Parâmetro & Grupo & Controle & Espontânea & 20 min PCV & 40 min PCV & 60 min PCV \\
\hline PAM (mmHg) & GI & $57.4 \pm 13.8$ & $47.6 \pm 15.94$ & $42 \pm 11.8$ & $46.8 \pm 17.7$ & $44.4 \pm 17.55$ \\
& GII & $43.8 \pm 13.79$ & $48.4 \pm 6.95$ & $51.4 \pm 9.5$ & $40.8 \pm 6.45$ & $39.4 \pm 7.02$ \\
FC (bat.min) & GI & $179,4 \pm 18,3$ & $193,2 \pm 37,1$ & $184,6 \pm 43,1$ & $187,4 \pm 25,5$ & $198,1 \pm 17,84$ \\
& GII & $183,0 \pm 24,7$ & $169,4 \pm 15,7$ & $167,8 \pm 24,2$ & $173,0 \pm 18,6$ & $182,0 \pm 21,5$ \\
IS0 exp. (\%) & GI & $0,88 \pm 0,63$ & $0,76 \pm 0,08$ & $0,76 \pm 0,18$ & $1,02 \pm 0,42$ & $0,84 \pm 0,54$ \\
& GII & $1,40 \pm 0,79$ & $1,48 \pm 0,45$ & $0,70 \pm 0,23$ & $0,98 \pm 0,32$ & $0,78 \pm 0,16$ \\
TC ( ${ }^{\circ}$ C) & GI & $38,4 \pm 0,43$ & $38,6 \pm 0,47$ & $38,8 \pm 0,65$ & $38,6 \pm 0,56$ & $38,6 \pm 0,62$ \\
& GII & $38,2 \pm 0,97$ & $38,24 \pm 0,67$ & $38,18 \pm 0,74$ & $37,76 \pm 0,81$ & $38,6 \pm 0,88$ \\
$\mathrm{SpO}_{2}(\%)$ & GI & $96,2 \pm 1,92$ & $94,0 \pm 3,16$ & $97,4 \pm 1,14$ & $95,8 \pm 1,7$ & $97,4 \pm 0,89$ \\
& GII & $97,0 \pm 1,22$ & $94,6 \pm 3,20$ & $97,4 \pm 1,14$ & $96,4 \pm 1,94$ & $96,6 \pm 1,34$ \\
\hline
\end{tabular}

* difere significativamente do valor da ventilação espontânea sendo $p<0,05$; \# difere significativamente do $G 20$ sendo $p<0,05$; Valores expressos em Média \pm DP

\section{DISCUSSÃO}

A falta de confiabilidade e de equipamentos de anestesia adequados para ventilação têm, historicamente, limitado o uso de técnicas de baixo fluxo em pediatria no nosso meio. Este conceito estende-se à anestesia veterinária em animais de pequeno porte, bem como em anestesia pediátrica "lato senso". Existem várias definições para baixo fluxo, uma delas refere-se à utilização de 0,5 a $1 \mathrm{~L} \cdot \mathrm{min}^{-1}$ de fluxo de gases frescos, enquanto que aquela com fluxo mínimo implica o uso de valores inferiores a 0,5 L. $\min ^{-11}$. Não se faz distinção por quilograma de peso. Recentemente, Tobin e col. ${ }^{2}$ avaliaram a eficiência de sistema circular com fole adulto para fornecer volume minuto em pulmão de teste infantil. Os autores demonstraram que quando o circuito circular adulto é usado na criança, a ventilação fornecida depende, fundamentalmente, da freqüência respiratória, pressão de pico inspiratória e complacência do pulmão sendo ventilado, mais do que o modo de ventilação usado, pressupondo que o uso de ventilador com circuito circular adulto na criança é factível. Igarashi e col. ${ }^{3}$ também demonstraram em crianças que o emprego de circuito circular de anestesia com baixo fluxo (600 $\mathrm{ml} \cdot \mathrm{min}^{-1}$ ) é muito viável. 
Tabela III - Parâmetros de Mecânica Respiratória dos Animais Submetidos à Pressão Inspiratória de 15 (GI) ou 20 cmH $\mathrm{cm}_{2}$ (GII) em Circuito com Reinalação de Anestesia em Diferentes Tempos de Avaliação

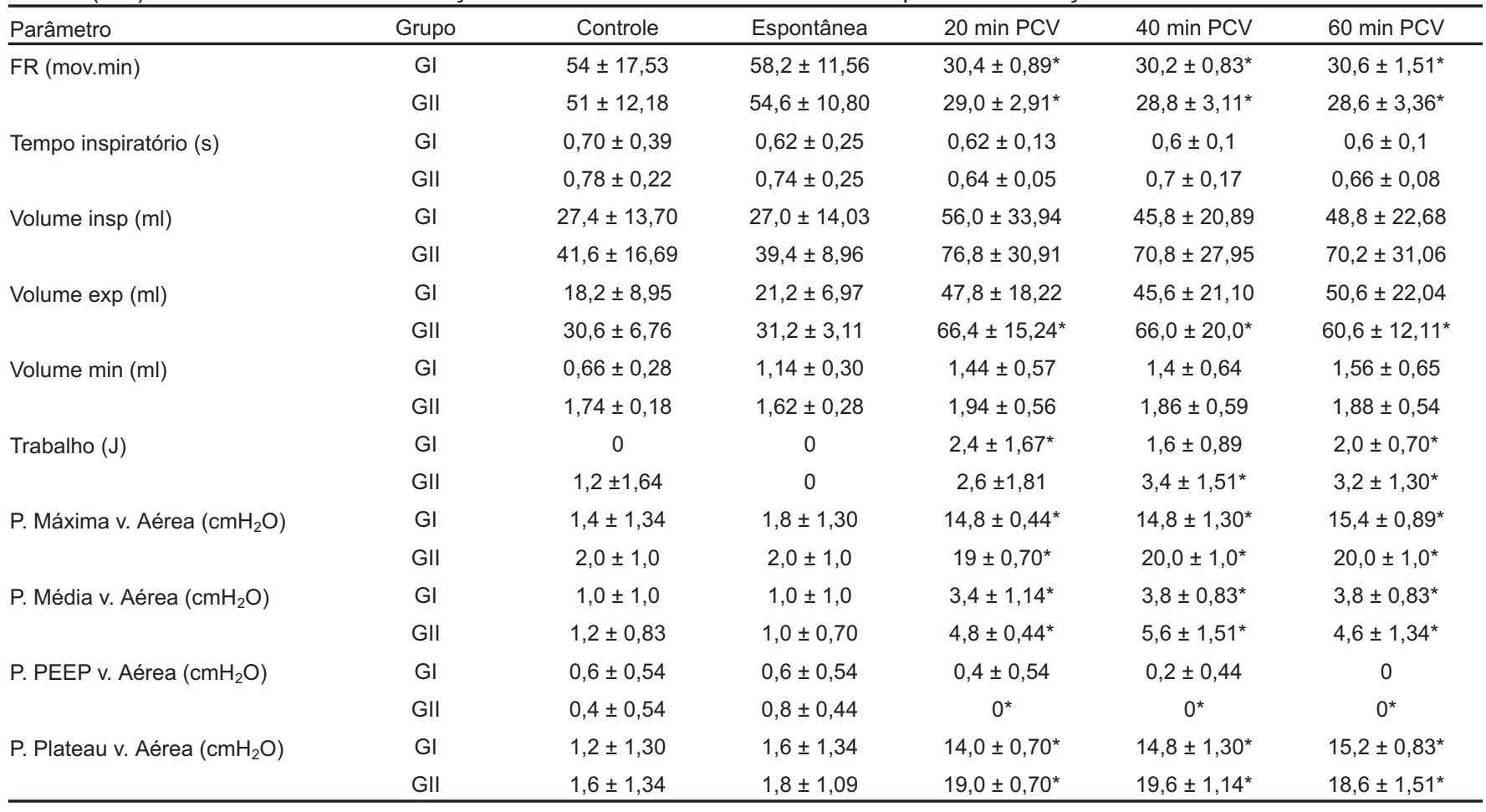

* difere significativamente do valor da ventilação espontânea sendo p<0,05; \# difere significativamente do G20 sendo p <0,05; Valores expressos em Média \pm DP

No presente estudo, o emprego de circuito circular fechado com pressão controlada permitiu adequada ventilação e oxigenação de indivíduos de peso baixo, sem o uso inconveniente de fluxos altos de gases frescos e consumo de pequena quantidade do anestésico inalatório.

A modalidade de ventilação empregada permitiu que se alcançassem volumes expirados altos e adequados (VE), mesmo com emprego de sonda traqueal de pequeno diâmetro e freqüência respiratória constante em 30 movimentos por minuto. Tobin e col. ${ }^{2}$ em estudo experimental, verificaram que no pulmão com complacência normal, o diâmetro da sonda, a freqüência respiratória empregada e o valor de PEEP são fatores limitantes para se alcançar adequados volumes expirados. Entretanto, no presente estudo, apesar do fato de somente os valores de PEEP variarem ao empregar-se sonda 3,5 com freqüência respiratória de 30 movimentos por minuto, alcançaram-se volumes adequados de VE, fato comprovado pelos parâmetros de ventilação obtidos no decorrer do estudo. Em ventilação, especialmente em pediatria, as pressões resistivas podem comprometer a ventilação alveolar, principalmente se associada a variações súbitas da complacência. $\mathrm{O}$ grupo de animais ventilados com $20 \mathrm{cmH}_{2} \mathrm{O}$ mostrou claramente este fato.

Conceitualmente em anestesia com sistema circular, a ventilação alveolar depende da força motriz gerada pelo "ventilador", sendo o fluxo de gases necessário apenas para carrear o agente anestésico e o oxigênio para as necessidades teciduais.

Revista Brasileira de Anestesiologia

Vol. 51, № 4, Julho - Agosto, 2001
De acordo com as propostas atuais de anestesia com baixos fluxos de gases frescos, no presente estudo realizou-se anestesia com fluxo mínimo ${ }^{1,4,5}$, tendo em vista o fato de se empregar $300 \mathrm{ml}$. $\mathrm{min}^{-1}$ de oxigênio. Apesar dos cálculos iniciais, nos quais se baseou a escolha do fluxo de gases empregados nas anestesias de baixo fluxo, advirem do consumo de oxigênio por quilograma de peso, nas definições da literatura não se leva em consideração o peso do paciente. Igarashi e col. ${ }^{3}$ usaram $600 \mathrm{ml}$. $\mathrm{min}^{-1}$ de gases frescos em pacientes pediátricos com peso médio de 16,4 kg para estudar a anestesia com fluxo baixo e sevoflurano, e Perkins e col. ${ }^{6}$ utilizaram $800 \mathrm{ml} . \mathrm{min}^{-1}$ para crianças com peso médio de $15 \mathrm{~kg}$.

Várias vantagens da utilização de fluxos baixos de gases frescos podem ser citadas: redução da poluição da sala cirúrgica; maior umidificação e manutenção da temperatura dos gases inspirados, e; redução no consumo dos anestésicos usados ${ }^{1,4,5}$.

Baxter ${ }^{5}$ relatou redução em $25 \%$ dos custos da anestesia ao reduzir o fluxo de gases frescos para $1 \mathrm{~L}$. $\mathrm{min}^{-1}$. Perkins e col. ${ }^{6}$ observaram diminuição de aproximadamente $58 \%$ no gasto de isoflurano e Igarashi e col. ${ }^{3}$ verificaram que o montante de sevoflurano empregado foi $1 / 7$ do total usado durante anestesia com fluxos convencionais de oxigênio $\left(6 \mathrm{~L}\right.$. $\left.\mathrm{min}^{-1}\right)$. No presente estudo, o consumo de isoflurano foi de $2 \mathrm{ml}$ para uma hora de anestesia. De acordo com Eger ${ }^{7}$, o uso crescente da nova geração de anestésicos inalatórios, sevoflurano e desflurano, deverá aumentar ainda mais a tendência de se 
utilizar as técnicas de baixo fluxo. Estes anestésicos, por possuírem baixa solubilidade, fazem com que o paciente absorva pequena quantidade do vapor anestésico. Também, devido a sua baixa potência, há a necessidade de se trabaIhar com pressões parciais elevadas no circuito de anestesia. Ao se usar altos fluxos de gases frescos, a maior parte do gás exalado é desperdiçada, sendo que elevadas concentrações do vapor anestésico terão que ser vaporizadas no circuito de anestesia para restabelecer as pressões parciais necessárias para a manutenção de plano anestésico adequado ${ }^{1,8}$. Por todos estes fatores, a única forma de se otimizar o uso destes novos agentes é empregando menor fluxo de gases frescos.

Na maior parte dos estudos que tratam da anestesia com baixo fluxo em crianças, os equipamentos de anestesia empregados são altamente sofisticados, o que certamente deve estar associado a um maior investimento por parte dos hospitais. No presente estudo, avaliou-se novo equipamento que se mostrou bastante seguro e de fácil manuseio na anestesia. Ao se despender menos na aquisição do equipamento, aliado à economia de anestésico, pode-se investir na compra de aparelhagem de monitorização de ventilação e gases anestésicos, mandatários para as anestesia com baixo fluxo de gases frescos.

Em conclusão, no presente estudo, a utilização do ventilador (VLL-5000), acoplado a fluxômetros e vaporizador convencional, mostrou-se eficiente e segura na troca gasosa, mantendo plano anestésico adequado na espécie sob investigação, permitindo inclusive a utilização de baixo fluxo de gases.

\section{AGRADECIMENTOS}

Os autores agradecem ao Dr. Humberto do Val pela cessão do equipamento para o experimento, bem como as informações técnicas sobre o mesmo.

\section{Controlled Pressure Mechanical Ventilation with Anesthesia Closed System for Low Weight Patients: Experimental Study}

Denise Tabacchi Fantoni, D.V.M., Sandra Mastrocinque D.V.M., Silvia Renata Gaido Cortopassi, D.V.M., Elton R. Migliatti,D.V.M., José Otávio Costa Auler Junior, M.D.

\section{INTRODUCTION}

There has been an increasing interest in the use of closed circuits in children in the last years. Such circuits associated to high precision ventilators allow for the use of lower fresh gas flows, better moisture and heat preservation and clearly less anesthetic consumption and operating room pollution ${ }^{1}$. An additional benefit is the possibility of using different ventilatory modalities and resources, such as positive end expiratory pressure (PEEP) available in such equipment, which are certainly needed for high risk patients. However, in our country, pediatric anesthesia has been a challenge for the anesthesiologist due to the lack of adequate equipment. In many centers, non-rebreathing circuits such as Bain's or Mapleson D systems are still widely use in pediatrics with manually controlled ventilation. These circuits use high fresh gas flows leading to high anesthetic consumption and environmental pollution. Another great disadvantage of such practice is the lack of accurate parameters monitoring, such as expired volume and airway pressure, among others.

Amajor drawback for the use of adult ventilators and anesthesia closed circuits, which could also be used for low weight patients, is their high cost. The possibility of low flow anesthesia in children would be another great advantage of this new equipment available in the market.

This study aimed at evaluating a closed circuit anesthesia machine using a pressure controlled time-cycled ventilatory mode with low fresh gas flow. Small animals (rabbits) were used to check the efficacy of this ventilator during anesthesia.

\section{METHODS}

Ten male and female rabbits weighing 3.5 to $5 \mathrm{~kg}$ were used. Anesthesia was induced with muscular xylazine $\left(10 \mathrm{mg} \cdot \mathrm{kg}^{-1}\right)$ and ketamine (25 mg. $\left.\mathrm{kg}^{-1}\right)$, followed by isoflurane under mask. Tracheal intubation was performed with Magill's cuffed tubes (2.5 to 3.0 of internal diameter). Anesthesia was maintained with isoflurane in $100 \%$ oxygen. Animals remained under spontaneous ventilation for 20 minutes until stabilization of the anesthesia machine parameters. Marginal artery and ear vein were catheterized for blood pressure monitoring as well as fluid ( $2 \mathrm{ml} . \mathrm{kg} \cdot \mathrm{h}^{-1}$ lactated Ringer's) and drugs administration, respectively. Pancuronium $(0.1$ $\mathrm{mg} . \mathrm{kg}^{-1}$ ) was then administered and time-cycled pressure controlled ventilation was installed for 60 minutes. Ventilator parameters were: respiratory rate of 30 movements per minute, I:E ratio of 1:2.5 and inspiratory time of 0.6 seconds.

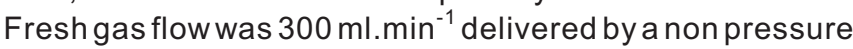
gauged flowmeter. Animals were then randomly distributed in two groups of five animals each. The ventilatory peak pressure was limited to $15 \mathrm{cmH}_{2} \mathrm{O}$ in Group I (G15) and $20 \mathrm{cmH}_{2} \mathrm{O}$ in Group II (G20). Ventilator gas flow was adjusted to generate a square pressure curve, compatible with the desired modality.

To evaluate adequate ventilation, expired air carbon dioxide concentration, $\mathrm{FiO}_{2}$ and hemoglobin peripheral oxygen saturation were continuously registered. Serial $\mathrm{pH}$, blood gases and plasma bicarbonate concentration were also measured. Using a ventilation monitor, inspired and expired volume, minute volume, peak, mean and plateau pressures, as well as pulmonary compliance were measured too. This monitor has 
assured respiratory modality through the visualization of pressure curves. To monitor anesthesia, inspired and expired isoflurane concentration, electrocardiogram and blood pressure were continuously evaluated.

A pneumatic time-cycled system with inspiratory and expiratory phase control was used for this study with the following adjustable controls: flow, inspiratory time, expiratory time, maximum pressure and PEEP, in addition to an alarm detecting disconnection or lack of cycle.

The system is made up of four sub-sets (Figure 1):

1. One time cycling subset with pressure limit;

2. One "Bag in a Bottle" system;

3. One subset made up of a combination of valves which allow the device to operate in controlled (manual or mechanical) or spontaneous ventilation, and;

4. One $\mathrm{CO}_{2}$ absorber reservoir.

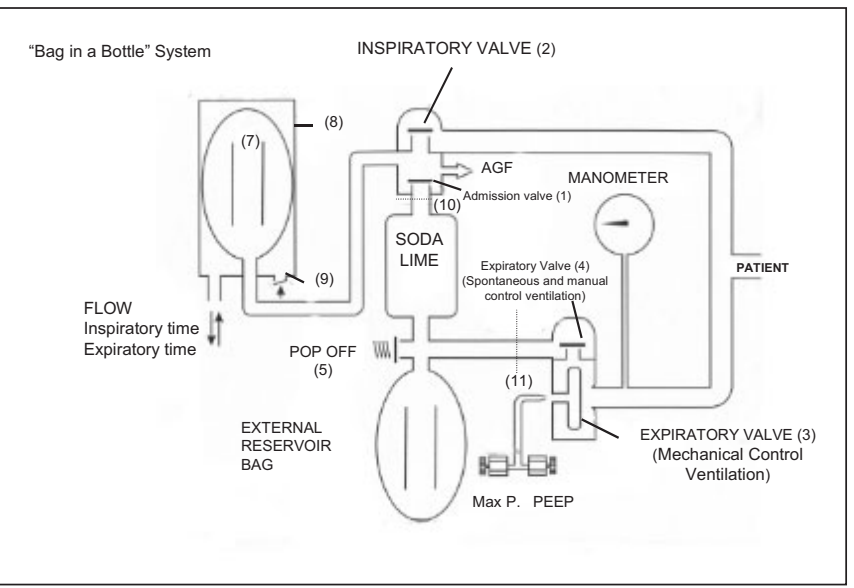

Figure 1 - System Used in Experiment

During the expiratory phase, a sub-atmospheric pressure acts between the internal reservoir bag (7) and the dome (8), allowing it to be filled with the gaseous mixture after going through the unidirectional valve (1). Another unidirectional valve (2) prevents the expired volume to return to the bag (7). During the inspiratory phase, a gas volume (generated by the flow control during inspiratory time) compresses the internal reservoir bag (7). The speed of this compression is a function of the flow set, which should mimic a manual compression. The unidirectional valve (1) closes and the valve (2) opens, allowing the bag's content (7) to reach the patient.

An expiratory valve (3) holds the tidal volume until the previously set airway pressure (maximum inspiratory pressure) is reached.

From this point on, the volume in excess escapes by opening and overcoming the unidirectional valve (4), and may go:

a) to the external reservoir bag (6); b) to the atmosphere or anti-pollution system through the pop-off valve (5), if the external reservoir bag (6) is full;

c) directly to the atmosphere if the system is open.

An additional gas flow (AGF) placed between valves (1) and (2) allows the second valve to be kept open, regardless of AGF and provided the internal reservoir bag (7) is full. This allows the small patient to ventilate without resistance. During the expiratory phase, the internal reservoir bag (7) is filled with the gaseous mixture coming from the external reservoir bag (6), as well as from the volume expired by the patient (after going through the $\mathrm{CO}_{2}$ absorber). If the system in use has no $\mathrm{CO}_{2}$ absorber, atmospheric air is then aspirated.

The valve (9) connects the external wall of the internal reservoir bag (7) to the atmosphere, and this happens with the equipment off (spontaneous and manually controlled ventilation). This valve also acts as a safety valve for the "Bag in a Bottle" system by preventing the pressure at this point to go beyond $80 \mathrm{~cm} \mathrm{H}_{2} \mathrm{O}$.

The expiratory branch was chosen to accurately measure mouth pressure, what was done before the valve (3). This measurement doesn't reflect the resistive pressure of flow going through the inspiratory way.

The configuration may be converted to a non $\mathrm{CO}_{2}$ absorbing system when one or both corrugated tubes (10 and 11), which connect the ventilator to the absorbing canister, are disconnected. Such procedure may be performed when a faster emergence is desired.

The equipment allows intermittent mandatory ventilation, provided that expiratory time be increased.

Results obtained were analyzed by a computer system (INSTAT), being data submitted to ANOVAanalysis of variance and Student's t test to compare means of both groups. Significance level of $5 \%$ was established.

\section{RESULTS}

Results are shown in tables I, II and III. There were no statistically significant differences between both experimental groups as to control and spontaneous ventilation moments. During spontaneous ventilation, high inspired and expired $\mathrm{CO}_{2}$ values were observed in both groups. With controlled ventilation, there has been a ventilation increment, confirmed by $\mathrm{P}_{\mathrm{ET}} \mathrm{CO}_{2}$ normalization. Inspired $\mathrm{CO}_{2}$ decreased and remained in values compatible with an adequate ventilation. Airway pressures, inspired and expired volumes suffered significant increases during controlled ventilation as compared to spontaneous ventilation.

When comparing both experimental groups, the only significant differences were seen with $\mathrm{PaCO}_{2}, \mathrm{PvO}_{2}, \mathrm{PvCO}_{2}$ and venous $\mathrm{pH}$. The group ventilated with $20 \mathrm{cmH}_{2} \mathrm{O}$ had lower $\mathrm{PaCO}_{2}$ (figure 2) and higher $\mathrm{PvO}_{2}$ and $\mathrm{pH}$ values. As to heart rate and blood pressure, there was no significant difference between groups or between evaluations performed before and after controlled ventilation. 
Table I - Ventilation, Oxygenation and pH Parameters of Animals Submitted to 15 (GI) or 20 (GII) $\mathrm{cmH}_{2} \mathrm{O}$ of Inspiratory Pressure in an Anesthesia Rebreathing Circuit in Different Moments

\begin{tabular}{|c|c|c|c|c|c|c|}
\hline Parameter & Group & Control & Spontaneous & 20 min PCV & 40 min PCV & $60 \min \mathrm{PCV}$ \\
\hline \multirow[t]{2}{*}{ Inspired $\mathrm{CO}_{2}(\mathrm{mmHg})$} & $\mathrm{GI}$ & $19.2 \pm 6.38$ & $24.0 \pm 6.42$ & $3.6 \pm 1.34^{*} \#$ & $3.6 \pm 0.89^{*}$ & $4.4 \pm 1.6^{*}$ \\
\hline & GII & $15.0 \pm 4.41$ & $16.2 \pm 3.83$ & $2.6 \pm 2.07^{*}$ & $2.4 \pm 1.67^{*}$ & $2.4 \pm 1.67^{\star}$ \\
\hline \multirow[t]{2}{*}{$\mathrm{P}_{\mathrm{ET}} \mathrm{CO}_{2}(\mathrm{mmHg})$} & GI & $48.0 \pm 8.42$ & $52.2 \pm 8.34$ & $40.6 \pm 6.38$ & $37.0 \pm 5.24$ & $44.0 \pm 5.98$ \\
\hline & GII & $49.4 \pm 6.50$ & $48.4 \pm 7.95$ & $33.6 \pm 9.37$ & $32.0 \pm 8.63^{*}$ & $33.0 \pm 9.24$ \\
\hline \multirow[t]{2}{*}{$\mathrm{pH}$} & $\mathrm{GI}$ & & $7.30 \pm 0.12$ & $7.34 \pm 0.07$ & & $7.33 \pm 0.12$ \\
\hline & GII & & $7.38 \pm 0.09$ & $7.45 \pm 0.06$ & & $7.43 \pm 0.06$ \\
\hline \multirow[t]{2}{*}{$\mathrm{PaCO}_{2}(\mathrm{mmHg})$} & $\mathrm{GI}$ & & $50.0 \pm 9.92$ & $37.2 \pm 8.5$ & & $42.8 \pm 18.58$ \\
\hline & GII & & $46.6 \pm 9.6$ & $32.2 \pm 5.8^{*}$ & & $29.4 \pm 4.72^{*}$ \\
\hline \multirow[t]{2}{*}{$\mathrm{PaO}_{2}(\mathrm{mmHg})$} & $\mathrm{Gl}$ & & $295.4 \pm 49.8$ & $267.8 \pm 103.8$ & & $256.4 \pm 64.86$ \\
\hline & GII & & $306.8 \pm 81.51$ & $216.6 \pm 91.1$ & & $286.0 \pm 112.8$ \\
\hline \multirow[t]{2}{*}{$\mathrm{HCO}_{3}$ (mEq.L) } & $\mathrm{GI}$ & $24.0 \pm 7.17$ & & $20.0 \pm 7.41$ & & $21.2 \pm 7.79$ \\
\hline & GII & $25.8 \pm 4.08$ & & $22.0 \pm 4.84$ & & $21.4 \pm 5.59$ \\
\hline \multirow[t]{2}{*}{ Venous $\mathrm{pH}$} & $\mathrm{GI}$ & $7.30 \pm 0.07$ & & $7.31 \pm 0.06$ & & $7.29 \pm 0.13$ \\
\hline & GII & $7.34 \pm 0.06$ & & $7.34 \pm 0.12$ & & $7.39 \pm 0.03$ \\
\hline \multirow[t]{2}{*}{$\mathrm{PvO}_{2}(\mathrm{mmHg})$} & $\mathrm{GI}$ & $74.0 \pm 44.4$ & & $64.2 \pm 16.7$ & & $53.6 \pm 5.68$ \\
\hline & GII & $61.0 \pm 18.66$ & & $72.0 \pm 42.98$ & & $63.6 \pm 37.46$ \\
\hline \multirow[t]{2}{*}{$\mathrm{PvCO}_{2}(\mathrm{mmHg})$} & $\mathrm{GI}$ & $44.4 \pm 17.37$ & & $34.2 \pm 10.2$ & & $44.4 \pm 21.19$ \\
\hline & GII & $36.0 \pm 14.0$ & & $29.4 \pm 5.45$ & & $31.0 \pm 2.34$ \\
\hline \multirow[t]{2}{*}{$\mathrm{SvO}_{2}(\%)$} & $\mathrm{GI}$ & $82.0 \pm 6.55$ & & $90.2 \pm 9.14$ & & $76.0 \pm 5.33$ \\
\hline & GII & $84.8 \pm 7.82$ & & $83.0 \pm 13.05$ & & $79.6 \pm 11.30$ \\
\hline
\end{tabular}

* significantly different from spontaneous ventilation ( $p<0.05)$; \# significantly different from G20 ( $<<0.05)$; Values expressed in Mean \pm SD

Table II - Cardiovascular and Anesthesia Parameters of Animals Submitted to 15 (GI) or $20 \mathrm{cmH}_{2} \mathrm{O}$ (GII) Inspiratory Pressure in an Anesthesia Rebreathing Circuit during Different Evaluation Times

\begin{tabular}{|c|c|c|c|c|c|c|}
\hline Parameter & Group & Control & Spontaneous & 20 min PCV & 40 min PCV & $60 \min \mathrm{PCV}$ \\
\hline \multirow[t]{2}{*}{$\mathrm{MBP}(\mathrm{mmHg})$} & $\mathrm{Gl}$ & $57.4 \pm 13.8$ & $47.6 \pm 15.94$ & $42 \pm 11.8$ & $46.8 \pm 17.7$ & $44.4 \pm 17.55$ \\
\hline & GII & $43.8 \pm 13.79$ & $48.4 \pm 6.95$ & $51.4 \pm 9.5$ & $40.8 \pm 6.45$ & $39.4 \pm 7.02$ \\
\hline \multirow[t]{2}{*}{ HR (beat.min) } & $\mathrm{Gl}$ & $179.4 \pm 18.3$ & $193.2 \pm 37.1$ & $184.6 \pm 43.1$ & $187.4 \pm 25.5$ & $198.1 \pm 17.84$ \\
\hline & GII & $183.0 \pm 24.7$ & $169.4 \pm 15.7$ & $167.8 \pm 24.2$ & $173.0 \pm 18.6$ & $182.0 \pm 21.5$ \\
\hline \multirow[t]{2}{*}{ Exp. ISO (\%) } & $\mathrm{GI}$ & $0.88 \pm 0.63$ & $0.76 \pm 0.08$ & $0.76 \pm 0.18$ & $1.02 \pm 0.42$ & $0.84 \pm 0.54$ \\
\hline & GII & $1.40 \pm 0.79$ & $1.48 \pm 0.45$ & $0.70 \pm 0.23$ & $0.98 \pm 0.32$ & $0.78 \pm 0.16$ \\
\hline \multirow[t]{2}{*}{$\mathrm{BT}\left({ }^{\circ} \mathrm{C}\right)$} & $\mathrm{Gl}$ & $38.4 \pm 0.43$ & $38.6 \pm 0.47$ & $38.8 \pm 0.65$ & $38.6 \pm 0.56$ & $38.6 \pm 0.62$ \\
\hline & GII & $38.2 \pm 0.97$ & $38.24 \pm 0.67$ & $38.18 \pm 0.74$ & $37.76 \pm 0.81$ & $38.6 \pm 0.88$ \\
\hline \multirow[t]{2}{*}{$\mathrm{SpO}_{2}(\%)$} & $\mathrm{GI}$ & $96.2 \pm 1.92$ & $94.0 \pm 3.16$ & $97.4 \pm 1.14$ & $95.8 \pm 1.7$ & $97.4 \pm 0.89$ \\
\hline & GII & $97.0 \pm 1.22$ & $94.6 \pm 3.20$ & $97.4 \pm 1.14$ & $96.4 \pm 1.94$ & $96.6 \pm 1.34$ \\
\hline
\end{tabular}

* significantly different from spontaneous ventilation ( $p<0.05$ ); \# significantly different from G20 ( $p<0.05$ ); Values expressed in Mean \pm SD

During controlled ventilation, isoflurane consumption was 2 $\mathrm{ml}$ per animal.

After 90 minutes of anesthesia, isoflurane was withdrawn and animals were extubated uneventfully.

\section{DISCUSSION}

The lack of adequate and reliable anesthesia ventilation equipment has historically limited the use of pediatric low flow techniques in our country. This concept is also applied to veterinary anesthesia in small animals as well to "latu sensu" pediatric anesthesia. There are several definitions to low flow anesthesia. One of them refers to the use of 0.5 to 1 L. $\mathrm{min}^{-1}$ of fresh gas flow, while minimum flow implies the use of values below $0.5 \mathrm{~L} \cdot \mathrm{min}^{-11}$. There is no distinction regarding body weight. Recently, Tobin et al. ${ }^{2}$ have evaluated the efficacy of a closed system with adult bellows to provide minute volume to pediatric test lung. Authors have shown that when an adult closed system is used in children, ventilation depends basically on respiratory rate, inspiratory peak pressure and lung compliance rather than the ventilation mode, sugVol. 51, No 4, Julho - Agosto, 2001 
Table III - Ventilatory Mechanics Parameters of Animals Submitted to 15 (GI) or $20 \mathrm{cmH}_{2} \mathrm{O}$ (GII) Inspiratory Pressure in Anesthesia Rebreathing Circuit during Different Evaluation Times

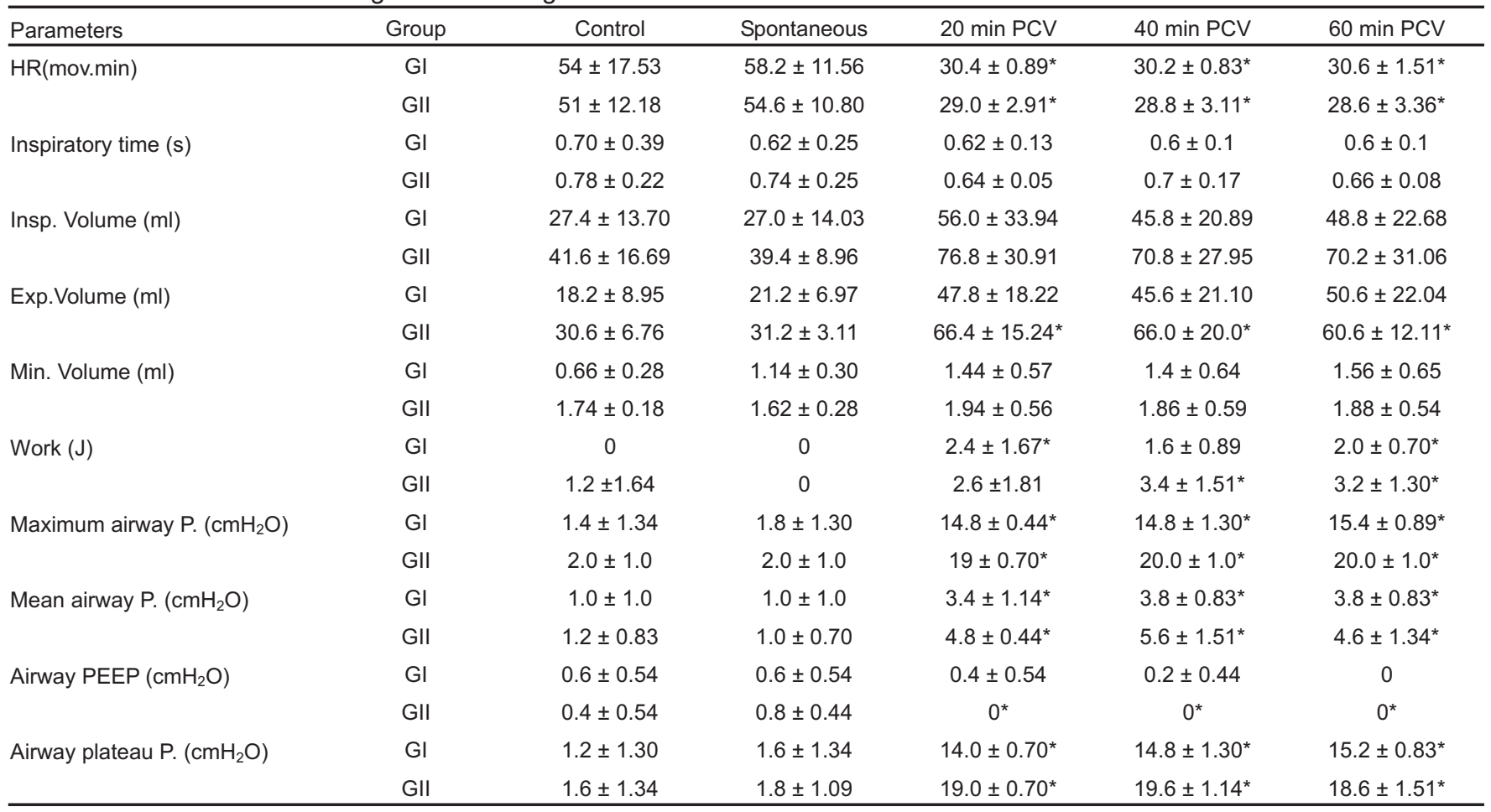

* significantly different from spontaneous ventilation $(p<0.05)$; \# significantly different from G20 ( $<<0.05)$; Values expressed in Mean \pm SD

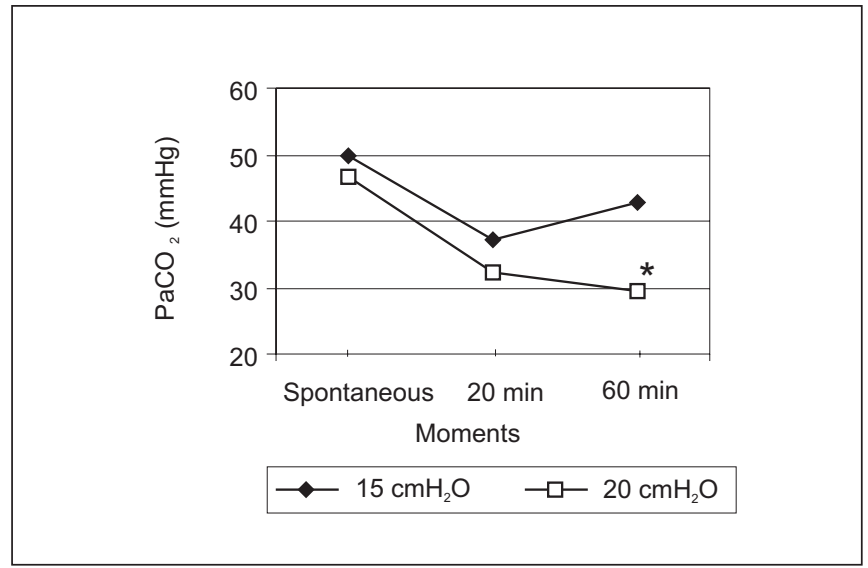

Figure 2 - Arterial Carbon Dioxide Partial Pressure $(\mathrm{mmHg})$ during Spontaneous Ventilation and at 20 and 60 Minutes of Controlled Ventilation in Groups Ventilated with 15 or $20 \mathrm{cmH}_{2} \mathrm{O}$

* significantly differente from group ventilated with $15 \mathrm{cmH}_{2} \mathrm{O}$

gesting that it is feasible to use an adult closed system for pediatric patients. Igarashi et al. ${ }^{3}$ have also shown that the use of a closed low flow anesthesia system $\left(600 \mathrm{ml}^{\mathrm{min}} \mathrm{m}^{-1}\right)$ is very feasible in children.

In our study, the pressure controlled closed system allowed adequate ventilation and oxygenation of low weight individuals without the inconvenience of high fresh gas flows and with low consumption of inhalational anesthetics.

Revista Brasileira de Anestesiologia

Vol. 51, № 4, Julho - Agosto, 2001
The ventilation modality provided high and adequate expired volumes (EV), even with narrow tracheal tubes and constant respiratory rate of 30 movements per minute. Tobin et al. ${ }^{2}$, in an experimental study, have observed that in a normal compliance lung, tube diameter, respiratory rate and PEEP values are limiting factors to obtain adequate expired volumes. In our study, however, despite PEEP values variations with the use of a 3.5 tube and a respiratory rate of 30 movements per minute, adequate EV volumes were reached, confirmed by ventilation parameters seen throughout the study. In ventilation, especially pediatric one, resistive forces may impair alveolar ventilation, especially if associated to sudden compliance variations. Animals ventilated with $20 \mathrm{~cm} \mathrm{H}_{2} \mathrm{O}$ have clearly shown such fact.

By concept, lung ventilation in closed system anesthesia depends on the driving force generated by the "ventilator", and the hole of gas flow is to supply the anesthetic agent and oxygen to tissue needs.

According to current concepts of low fresh gas flow anesthesia, our study was performed with minimum flow anesthesia $1,4,5$, since we used $300 \mathrm{ml}$. $\mathrm{min}^{-1}$ of oxygen flow. To date, in spite of initial calculations in which the choice of gas flow used in low flow anesthesia is a function of oxygen consumption per kilogram, such definition does not take into account

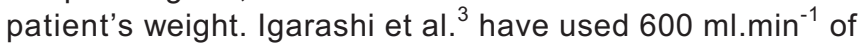
fresh gases in pediatric patients with mean weight of $16.4 \mathrm{~kg}$ to study low flow anesthesia with sevoflurane. Perkins et al. ${ }^{6}$ have used $800 \mathrm{ml} . \mathrm{min}^{-1}$ in children with mean weight of $15 \mathrm{~kg}$. 
There are several advantages in the use of low fresh gas flows: decreased operating room pollution, better moisture and temperature maintenance of inspired gases and lower anesthetic consumption ${ }^{1,4,5}$.

Baxter ${ }^{5}$ has reported a $25 \%$ anesthesia costs decrease with fresh gas flow reduction to at $1 \mathrm{~L} \cdot \mathrm{min}^{-1}$. Perkins et al. ${ }^{6}$ have observed an approximately $58 \%$ decrease in isoflurane consumption while Igarashi et al. ${ }^{3}$ have noticed that sevoflurane consumption was $1 / 7$ of the total used during anesthesias with conventional oxygen flows ( 6 L. $\left.\mathrm{min}^{-1}\right)$. In our study, isoflurane consumption was $2 \mathrm{ml}$ for one-hour anesthesia. According to Eger $^{7}$, the increasing use of the new generation inhalational anesthetics, sevoflurane and desflurane, shall increase even more the use of low flow techniques. For having a low solubility, such anesthetics are absorbed in smaller amounts. Also, due to their lower potency, high partial pressures in the anesthesia circuit are needed. With high fresh gas flows, most exhaled gas is wasted, and a great amount of anesthetics has to be vaporized and delivered into the anesthesia circuit to re-establish high partial pressures needed to maintain an adequate anesthesia depth ${ }^{1,8}$. For all these reasons, the only way to optimize the use of the new agents is to use a lower fresh gas flow.

In most studies dealing with low flow anesthesia in children, anesthesia machines are highly sophisticated, what must be related to greater hospital investments. Our study has evaluated a new equipment which has shown to be very safe and easy to use. Spending less with equipment and using less anesthetic drugs, it is possible to invest in the purchase of ventilation and anesthetic gases monitoring devices, mandatory for low fresh gas flow anesthesia.

In conclusion, in our study, the use of the ventilator (VLL-5000) coupled to conventional flowmeters and vaporizers was efficient and safe in gaseous exchange, maintaining an adequate anesthesia depth in the species under investigation and allowing the use of low gas flows.

\section{ACKNOWLEDGEMENTS}

The authors thank Dr. Humberto do Val for lending the equipment for the experiment, as well as for supplying technical information on it.

\section{REFERÊNCIAS - REFERENCES}

01. Baum JA - Low-flow anaesthesia. Eur J Anaesthesiol, 1996;13:432-435.

02. Tobin MJ, Stevenson GW, Horn BJ e al - A comparison of three modes of ventilation with the use of an adult circle system in an infant lung model. Anesth Analg, 1998;87:766-771.
03. Igarashi $\mathrm{M}$, Watanabe $\mathrm{H}$, Iwasaki $\mathrm{H}$ et al - Clinical evaluation of low-flow sevoflurane anaesthesia for paediatric patients. Acta Anaesthesiol Scand, 1999;43:19-23.

04 . Baker AB - Low flow and closed circuits. Anaesth Intensive Care, 1994;22:341-342.

05. Baxter AD - Low and minimal flow inhalational anaesthesia. Can J Anaesth, 1997;44:643-653.

06. Perkins R, Meakin G - Economics of low-flow anaesthesia in children. Anaesthesia, 1996;51:1089-1092.

07. Eger El - Economic analysis and pharmaceutical policy: a consideration of the economics of the use of desfluorane. Anaesthesia, 1995;50:45-48.

08. Auler Jr JOC, Carmona MJ, Barbas CV et al - The effects of positive end-expiratory pressure on respiratory system mechanics and hemodynamics in postoperative cardiac surgery patients. Braz J Med Biol Res, 2000;33:31-42.

\section{RESUMEN}

Fantoni DT, Mastrocinque S, Cortopassi SRG, Migliatti ER, Auler Jr JOC - Empleo de la Ventilación Mecánica con Presión Controlada en Circuito Circular de Anestesia para Pacientes de Bajo Peso: Estudio Experimental

Justificativa y Objetivos - La anestesia con bajo flujo, en pacientes pediátricos, requiere equipamientos adecuados siendo caros los disponibles, y limitando su uso. Este estudio evaluó la anestesia con bajo flujo en conejos, empleando circuito cerrado, modo de presión controlada en nuevo ventilador pediátrico para anestesia (VPL-5000A-Vent-Logos).

Método - Diez conejos fueron distribuidos aleatoriamente, siendo que el grupo I fue sometido a presión de $15 \mathrm{cmH}_{2} \mathrm{O}$ y el grupo II a la de $20 \mathrm{cmH}_{2} \mathrm{O}$. La anestesia fue realizada con xilazina $\left(10 \mathrm{mg} \mathrm{kg}^{-1}\right)$ y cetamina $\left(25 \mathrm{mg} \cdot \mathrm{kg}^{-1}\right)$ asociados, por vía muscular, seguida de manutención con isoflurano, después intubación orotraqueal. Después de 20 minutos, se administró pancuronio $\left(0,1 \mathrm{mg} \cdot \mathrm{kg}^{-1}\right)$ por vía venosa y la ventilación controlada fue iniciada. Los parámetros ajustados y el ventilador fueron: FR $30 \mathrm{mpm}$, frecuencia I:E 1:2,5 y tiempo de inspiración 0, 6 segundo, además de las presiones de plateau. El flujo de gases frescos empleado fue de $300 \mathrm{ml}$ (total). Los parámetros fueron colectados a cada 20 minutos durante una hora. Los datos obtenidos fueron sometidos a análisis estadística de variancia para medidas repetidas $(p<0,05)$.

Resultados - El $\mathrm{CO}_{2}$ reinhalado diminuyó significativamente en el grupo II, de $15 \mathrm{mmHg}$, durante la ventilación espontanea, para un valor medio de $2,4 \mathrm{mmHg}$ durante a ventilación controlada. En el grupo l, de 19,2 $\mathrm{mmHg}$ (inicial) para 3,6 $\mathrm{mmHg}$. Comparándose los dos grupos, diferencias significativas fueron encontradas en relación al $\mathrm{pH}$ venoso, $\mathrm{PaCO}_{2}, \mathrm{PVO}_{2}$ y discreta diferencia entre la PAM y PAD. El grupo de $15 \mathrm{cmH}_{2} \mathrm{O}$ presentó importante acidosis respiratoria, en cuanto el de $20 \mathrm{cmH}_{2} \mathrm{O}$ obtuvo valores normales de $\mathrm{pH} y$ $\mathrm{PaCO}_{2}$. Una vez que los valores de volumen expirado entre los grupos fueron semejantes, tales diferencias entre $\mathrm{pH}$ y gases sangüíneos presentados por los grupos pueden estar relacionadas a los bajos niveles de $\mathrm{pH}$ observados en el grupo I. Se Verificó consumo medio de $2 \mathrm{ml}$ de isoflurano por animal durante los 120 minutos de estudio.

Conclusiones - Con equipamiento adecuado es posible emplear anestesia de bajo flujo, ventilación con presión controlada y circuito cerrado en pacientes con muy bajo peso. 